\title{
GlycA, a novel biomarker of systemic inflammation and cardiovascular disease risk
}

\author{
Margery A. Connelly ${ }^{1}$, James D. Otvos ${ }^{1}$, Irina Shalaurova ${ }^{1}$, Martin P. Playford ${ }^{2}$ and Nehal N. Mehta2*
}

\begin{abstract}
Background: GlycA is a novel spectroscopic marker of systemic inflammation with low intra-individual variability and other attributes favoring its clinical use in patients with chronic inflammatory and autoimmune diseases. GlycA is unique in its composite nature, reflecting both increased glycan complexity and circulating acute phase protein levels during local and systemic inflammation. Recent studies of GlycA from cross-sectional, observational and interventional studies have been highly informative, demonstrating that GlycA is elevated in acute and chronic inflammation, predicts death in healthy individuals and is associated with disease severity in patients with chronic inflammatory diseases such as rheumatoid arthritis, psoriasis and lupus. Moreover, following treatment with biological therapy in psoriasis, reduction in skin disease severity was accompanied by a decrease in GlycA levels and improvement in vascular inflammation.

Conclusions: Collectively, these findings suggest GlycA is a marker that tracks systemic inflammation and subclinical vascular inflammation. However, larger prospective studies and randomized trials are necessary in order to assess the impact of novel therapies on GlycA in patients with chronic inflammatory conditions, which may be concomitant with cardiovascular benefits.
\end{abstract}

Keywords: Nuclear magnetic resonance spectroscopy, GlycA, Inflammation, Cardiovascular disease

\section{GlycA is a clinical biomarker of systemic inflammation}

GlycA is the name given to a particular inflammationresponsive signal in proton $\left({ }^{1} \mathrm{H}\right)$ nuclear magnetic resonance (NMR) spectra of serum and plasma measured clinically [1]. The GlycA NMR signal arises largely from the $N$-acetyl methyl group protons of mobile $\mathrm{N}$-acetyl glucosamine residues on the glycan portions of acute-phase proteins such as $\alpha 1$-acid glycoprotein (orosomucoid), haptoglobin, $\alpha 1$-antitrypsin and $\alpha 1$-antichymotrypsin [1-3]. GlycA levels, in units of $\mu \mathrm{mol} / \mathrm{L}$ glycoprotein $\mathrm{N}$-acetyl methyl groups, are associated with concentrations of IL-6, tumor necrosis factor (TNF)- $\alpha$, fibrinogen, C-reactive protein (CRP; hsCRP as assessed by high-sensitivity assay), serum amyloid A (SAA), and lipoprotein-associated phospholipase $A_{2}$

\footnotetext{
*Correspondence: nehal.mehta@nih.gov

${ }^{2}$ National Heart, Lung, and Blood Institute, National Institutes of Health, Bethesda, MD 20892, USA

Full list of author information is available at the end of the article
}

(Lp-PLA 2 ) [1, 3-9]. Moreover, GlycA levels are associated with increased production of anti-microbial peptides, circulating leukocytes and neutrophil activity [3, 10]. In fact, two of the major protein contributors to the GlycA signal, $\alpha 1$-acid glycoprotein and haptoglobin, are synthesized in and secreted from neutrophil granules, suggesting that, besides the liver, neutrophils may be a relevant source of elevated GlycA [3, 10]. However, CRP, SAA and cytokines circulate at much lower concentrations than $\alpha 1$-acid glycoprotein, haptoglobin, and $\alpha 1$-antitrypsin, and are not highly glycosylated, therefore they contribute negligibly to the measured GlycA signal [1]. Reduced glycan mobility is another reason why some proteins with $\mathrm{N}$-acetyl glucosamine residues, such as fibrinogen and immunoglobulin G (IgG), do not produce observable glycan NMR signals [1]. Since both positive acute phase protein levels and glycan complexity increase during inflammation, GlycA is higher in patients with acute febrile illnesses as well as chronic inflammatory diseases $[3,10,11]$. 


\section{GlycA is a marker of cardiometabolic risk}

GlycA is positively correlated with body mass index (BMI), insulin resistance, markers of metabolic syndrome and the ratio of leptin to adiponectin, suggesting that in addition to being elevated in acute inflammation, levels are also raised along with adipose tissue-associated lowgrade chronic inflammation [7, 12]. As such, GlycA may be a reliable biomarker of cardiometabolic risk [13]. In support of this concept, it has been reported that GlycA is associated with the presence and extent of coronary artery disease (CAD) in a secondary prevention cardiovascular (CV) cohort (CATHGEN) [14] and with incident CV events in the Women's Health Study (WHS) [15], PREVEND [16], MESA [17] and JUPITER trial [18], independent of traditional CV disease risk factors. Notably, these associations were usually only slightly attentuated, if at all, by hsCRP implying that GlycA measures $\mathrm{CV}$ disease risk beyond hsCRP. GlycA is also associated with incident type 2 diabetes mellitus (T2DM) [19, 20]. Of note, the association of GlycA with incident T2DM remained statistically significant both in the WHS and PREVEND even after adjusting for traditional diabetes risk factors and hsCRP $[19,20]$. Taken together, evidence suggests that GlycA may also serve as a useful biomarker for assessment of $\mathrm{CV}$ disease risk as well as risk of progression to T2DM.

\section{GlycA is associated with disease activity and CV disease in chronic inflammatory diseases}

GlycA concentrations are higher in patients with autoimmune diseases such as rheumatoid arthritis (RA) [5, 21], systemic lupus erythematosus (SLE) $[22,23]$ and psoriasis [24]. In a cross-sectional study, GlycA was higher in RA patients compared to control subjects and was associated with increasing degree of RA disease activity $[5,21]$. In this study, GlycA significantly correlated with Larsen score, a radiographic scoring of joint disease, whereas hsCRP and erythrocyte sedimentation rate (ESR) did not [5]. Moreover, GlycA was associated with coronary calcium score and known CAD in patients with RA [5]. GlycA levels were also higher in patients with SLE than matched control subjects [22]. In a crosssectional analysis, GlycA levels were positively associated with ESR, hsCRP, E-selectin, sICAM-1 and triglycerides (TG), but not with creatinine, SLE Disease Activity Index (SLEDAI) or coronary calcium score [22]. In a separate SLE cohort, mean GlycA levels were somewhat higher in patients with high disease activity vs. patients with low or no disease activity [23]. In a longitudinal analysis, GlycA increased significantly along with increased SLEDAI [23]. Furthermore, a study in two cohorts of patients with psoriasis revealed that GlycA increased with psoriasis skin disease severity as measured by the Psoriasis
Area Severity Index (PASI) [24]. GlycA was associated with non-calcified and total CAD plaque burden, as well as aortic vascular inflammation, in patients with psoriasis, independent of traditional $\mathrm{CV}$ risk factors including hsCRP [24]. The latter was true even after anti-TNF treatment [24]. On the other hand, after adjustment, hsCRP was not associated with total CAD or vascular inflammation in patients with psoriasis [24]. Taken together, these data support the concept that GlycA is a marker of disease activity and subclinical $\mathrm{CV}$ disease in patients with RA and psoriasis.

As in patients with T2DM, it is well known that patients with RA and psoriasis have higher risk of $\mathrm{CV}$ disease and CV events [25-31]. Traditional risk factors such as low density lipoprotein cholesterol (LDL-C) and hsCRP alone are not consistent predictors of $\mathrm{CV}$ disease risk in these patients. For example, LDL-C is often in the normal range, despite higher $\mathrm{CV}$ disease risk in $\mathrm{RA}$ patients [32-34]. This is often dubbed the "lipid paradox" of RA [32-34]. Moreover, addition of hsCRP to both the Framingham Risk Score (FRS) and QRISK2 equations was not associated with a significant improvement in reclassification [35]. Due to its association with CV disease in autoimmune disease patients, GlycA may represent a more effective means for assessing $\mathrm{CV}$ disease risk in patients with RA and psoriasis [5, 24], which remains an unmet medical need as RA and psoriasis patients continue to be at high risk of $\mathrm{CV}$ events and cardiovascular related mortality despite therapy [25-31].

\section{GlycA as a marker of mortality or prognosis}

Besides being a marker of CV and T2DM risk, GlycA is also associated with mortality. Similar to IL-6 and D-dimer, GlycA was found to be related to all-cause death in MESA [17]. GlycA was also associated with colorectal cancer incidence and colorectal cancer mortality, but not breast cancer or mortality from any other cancer in the WHS [36]. GlycA was broadly predictive of hospitalization from infection and infection related mortality, even after adjusting for prevalent $\mathrm{CV}$ disease, diabetes, cancer, immunodeficiencies and obesity [3]. In CATHGEN, a cohort of patients undergoing cardiac catheterization, GlycA was associated with all-cause mortality, CV mortality and non-CV mortality post-catheterization [14].

\section{Differences between GlycA and hsCRP}

As a composite biomarker, GlycA may be a better reflection of a systemic acute phase response than any single glycoprotein component [1]. For example, assays for measuring individual acute phase proteins, such as hsCRP, often exhibit high intra-individual variability [37-40]. One approach to overcome this issue is to acquire several measures of an individual marker and use 
the average value to assess inflammation-related risk. A second approach is to measure multiple inflammatory markers at once. GlycA, however, is already a composite biomarker that integrates the protein levels and glycosylation states of several of the most abundant acute phase proteins in serum. This allows for a more stable measure of inflammation with lower intra-individual variability for GlycA than hsCRP $[1,11]$. Consequently, while guidelines recommend two serial measurements be taken at least 2 weeks apart when using hsCRP for $\mathrm{CV}$ risk assessment, only one measurement is necessary for evaluation of a patient's CV risk using the GlycA test [11].

Despite similarities in disease associations, GlycA and hsCRP likely capture different aspects of the inflammatory response [10]. Epidemiological evidence for this speculation stems from the associations between GlycA and incident CV events or T2DM which were often only slightly attenuated, if at all, by the addition of hsCRP to the regression model. These results imply that GlycA measures CVD and T2DM risk beyond hsCRP [14-20]. This was also observed in the published RA study where GlycA levels, but not hsCRP, correlated with radiographic damage as measured by the Larsen score [5] as well as in the psoriasis study where GlycA, but not hsCRP, was associated with aortic vascular inflammation after adjusting for traditional CV risk factors [24]. In addition, baseline levels of both GlycA and hsCRP were found to be independent and additive markers of risk for future major adverse cardiac events (MACE), especially death and heart failure hospitalization, in patients who underwent angiography for CAD [41]. Furthermore, in most clinical studies the correlations between GlycA and hsCRP are between 0.20 and 0.50 , suggesting that there is some overlap in biological processes they are detecting. This is not surprising given that CRP is an early acute phase response protein and the proteins that contribute the most to the GlycA signal ( $\alpha 1$-acid glycoprotein, haptoglobin and $\alpha 1$-antitrypsin) rise later in the acute phase response [42].

\section{Clinical utility of the GlycA test and future perspectives}

Based on the evidence gathered to date, GlycA test results may have clinical utility similar or complementary to hsCRP, fibrinogen and other biomarkers of inflammation [1, 14-20]. Therefore, GlycA may be used for the following intended uses: (1) as an aid in the identification and stratification of individuals at risk for future $\mathrm{CV}$ disease, (2) as an independent marker of prognosis for recurrent $\mathrm{CV}$ events in patients with stable coronary disease or acute coronary syndrome (ACS), (3) as an aid in the assessment of disease activity and risk of $\mathrm{CV}$ disease in adult RA and psoriasis patients when used in conjunction with standard clinical assessment and (4) in the evaluation of risk of progression to T2D along when considering measures of insulin resistance [43].

Of note, questions have arisen as to why "GlycA" values measured by a high-volume NMR metabolomics research platform [3, 10, 44, 45] are three- to fourfold higher than those produced by the LabCorp "clinical" GlycA assay. The origin of these differences is likely the failure of NMR metabolomics to completely separate the GlycA signal from overlapping NMR signals from proteins and very low density lipoprotein (VLDL) particles that carry the bulk of circulating triglycerides (TG). As a consequence, published correlations with TG levels are higher for the metabolomics measured GlycA $(r=0.69)$ than for hsCRP in the same study $(r=0.28)[45,46]$. Where TG correlations were reported for both hsCRP and "clinical GlycA", the correlations were lower in magnitude ( $\mathrm{r} \sim 0.2$ to 0.4 ) $[1,8,16,22]$. Adjustment for TG in multivariate regression models may alleviate some of this concern for epidemiological studies [3], but it would be difficult to use the "metabolomics GlycA" for clinical management of individual patients.

In addition to patient care applications, the potential exists for GlycA to be useful as a key biomarker in clinical studies and drug development programs. Research is underway to better characterize the relationship of inflammatory biomarkers and clinical response to various therapies. As such, GlycA measurements have been evaluated in a number of clinical trials. While GlycA levels were not significantly reduced by rosuvastatin treatment [18], they were reduced by anti-TNF therapy [24]. These studies highlight the fact that GlycA potentially provides unique information for assessing safety and monitoring efficacy of anti-inflammatory treatment. Moreover, the Phase III CANTOS study results revealed that treatment with the IL- $1 \beta$ antibody, canakinumab, in combination with standard of care therapy, reduced $\mathrm{CV}$ risk in people with a prior heart attack and inflammatory atherosclerosis [47]. This provides an additional opportunity to evaluate the relationship of various inflammatory biomarkers, including GlycA, with response to therapy. In both cases, future research may indicate the potential of GlycA to adjudicate individual response to therapy, as well as identify individuals who may benefit from further adjustment of treatment.

\section{Conclusions}

GlycA is a composite biomarker of systemic inflammation that integrates both the protein levels and glycosylation states of several of the most abundant acute phase proteins in serum or plasma. GlycA is higher in acute and chronic inflammatory conditions and is associated with disease severity in patients with rheumatoid arthritis, 
systemic lupus erythematosus and psoriasis. Despite similarities in disease associations, GlycA and hsCRP likely capture different aspects of the inflammatory response. GlycA is also a marker of cardiometabolic risk that is associated with body mass index, insulin resistance, incident CV disease events and T2DM. GlycA is associated with coronary artery calcium and known CAD in rheumatoid arthritis and active vascular inflammation in psoriasis patients. Therefore, GlycA is novel a marker of $\mathrm{CV}$ risk that may be useful in autoimmune patients where traditional risk factors and risk scores may not accurately capture CV risk.

\begin{abstract}
Abbreviations
ACS: acute coronary syndrome; BMI: body mass index; CAD: coronary artery disease; CANTOS: Canakinumab Anti-inflammatory Thrombosis Outcomes Study; CATHGEN: CATHeterization GENetics Study; CV: cardiovascular; hsCRP: high sensitivity C-reactive protein; ESR: erythrocyte sedimentation rate; FRS: Framingham risk score; JUPITER: Justification for the Use of Statins in Prevention: an Intervention Trial Evaluating Rosuvastatin; LDL-C: low density lipoprotein cholesterol; MACE: major adverse cardiac events; MESA: MultiEthnic Study of Atherosclerosis; NMR: nuclear magnetic resonance; PREVEND: Prevention of Renal and Vascular End-stage Disease Study; RA: rheumatoid arthritis; SAA: serum amyloid A; SLE: systemic lupus erythematosus; SLEDAI: SLE disease activity index; T2DM: type 2 diabetes mellitus; TG: triglycerides; TNF: tumor necrosis factor; VLDL: very low density lipoprotein; WHS: Women's Health Study.
\end{abstract}

\section{Authors' contributions}

All authors contributed to the writing of this review. All authors read and approved the final manuscript.

\section{Author details}

${ }^{1}$ Laboratory Corporation of America Holdings (LabCorp), Morrisville, NC 27560, USA. ${ }^{2}$ National Heart, Lung, and Blood Institute, National Institutes of Health, Bethesda, MD 20892, USA.

\section{Acknowledgements}

The authors would like to thank Dr. William C. Cromwell for comments and critical review of the manuscript.

\section{Competing interests}

MAC, JDO and IS are employees of LabCorp. Dr Nehal Mehta is a full time US Government Employee and has received research grants from Abbvie, Janssen, Celgene and Novartis. No other competing interests were declared by the authors.

\section{Availability of data and materials}

Data sharing not applicable to this article as no new datasets were generated or analyzed for the purpose of this review paper.

\section{Consent for publication}

Not applicable.

\section{Ethics approval and consent to participate}

Not applicable.

\section{Funding}

HL-006193-4 (Intramural Grant to NNM from the NHLBI).

\section{Publisher's Note}

Springer Nature remains neutral with regard to jurisdictional claims in published maps and institutional affiliations.

Received: 5 September 2017 Accepted: 17 October 2017
Published online: 27 October 2017

\section{References}

1. Otvos JD, Shalaurova I, Wolak-Dinsmore J, Connelly MA, Mackey RH, Stein $J H$, Tracy RP. GlycA: a composite nuclear magnetic resonance biomarker of systemic inflammation. Clin Chem. 2015;61:714-23.

2. Bell JD, Brown JC, Nicholson JK, Sadler PJ. Assignment of resonances for 'acute-phase' glycoproteins in high resolution proton NMR spectra of human blood plasma. FEBS Lett. 1987;215:311-5.

3. Ritchie SC, Wurtz P, Nath AP, Abraham G, Havulinna AS, Fearnley LG, Sarin AP, Kangas AJ, Soininen P, Aalto K, et al. The biomarker GlycA is associated with chronic inflammation and predicts long-term risk of severe infection. Cell Syst. 2015;1:293-301.

4. Dungan K, Binkley P, Osei K. GlycA is a novel marker of inflammation among non-critically ill hospitalized patients with type 2 diabetes. Inflammation. 2015;38:1357-63.

5. Ormseth MJ, Chung CP, Oeser AM, Connelly MA, Sokka T, Raggi P, Solus JF, Otvos JD, Stein CM. Utility of a novel inflammatory marker, GlycA, for assessment of rheumatoid arthritis disease activity and coronary atherosclerosis. Arthritis Res Ther. 2015;17:117.

6. Dullaart RP, Gruppen EG, Connelly MA, Lefrandt JD. A pro-inflammatory glycoprotein biomarker is associated with lower bilirubin in metabolic syndrome. Clin Biochem. 2015;48:1045-7.

7. Dullaart RP, Gruppen EG, Connelly MA, Otvos JD, Lefrandt JD. GlycA, a biomarker of inflammatory glycoproteins, is more closely related to the leptin/adiponectin ratio than to glucose tolerance status. Clin Biochem. 2015;48:811-4.

8. Gruppen EG, Connelly MA, Otvos JD, Bakker SJ, Dullaart RP. A novel protein glycan biomarker and LCAT activity in metabolic syndrome. Eur J Clin Invest. 2015;45:850-9.

9. Gruppen EG, Connelly MA, Dullaart RP. Higher circulating GlycA, a proinflammatory glycoprotein biomarker, relates to lipoprotein-associated phospholipase A2 mass in nondiabetic subjects but not in diabetic or metabolic syndrome subjects. J Clin Lipidol. 2016;10:512-8.

10. Ritchie SC, Wurtz P, Nath AP, Abraham G, Havulinna AS, Kangas AJ, Soininen P, Aalto K, Seppala I, Raitoharju E, et al. Systems medicine links microbial inflammatory response with glycoprotein-associated mortality risk. BioRxiv. 2015. doi:10.1101/018655.

11. Connelly MA, Shimizu C, Winegar DA, Shalaurova I, Pourfarzib R, Otvos JD, Kanegaye JT, Tremoulet AH, Burns JC. Differences in GlycA and lipoprotein particle parameters may help distinguish acute Kawasaki Disease from other febrile illnesses in children. BMC Pediatr. 2016;16:151.

12. Lorenzo C, Festa A, Hanley AJ, Rewers MJ, Escalante A, Haffner SM. Novel protein glycan-derived markers of systemic inflammation and C-reactive protein in relation to glycemia, insulin resistance, and insulin secretion. Diabetes Care. 2017;40(3):375-82.

13. Connelly MA, Gruppen EG, Otvos JD, Dullaart RP. Inflammatory glycoproteins in cardiometabolic disorders, autoimmune diseases and cancer. Clin Chim Acta. 2016;459:177-86.

14. McGarrah RW, Kelly JP, Craig DM, Haynes C, Jessee RC, Huffman KM, Kraus WE, Shah SH. A novel protein glycan-derived inflammation biomarker independently predicts cardiovascular disease and modifies the association of HDL subclasses with mortality. Clin Chem. 2017;63:288-96.

15. Akinkuolie AO, Buring JE, Ridker PM, Mora S. A novel protein glycan biomarker and future cardiovascular disease events. J Am Heart Assoc. 2014;3:e001221.

16. Gruppen EG, Riphagen IJ, Connelly MA, Otvos JD, Bakker SJ, Dullaart RP. GlycA, a pro-inflammatory glycoprotein biomarker, and incident cardiovascular disease: relationship with C-reactive protein and renal function. PLOS ONE. 2015;10:e0139057.

17. Duprez DA, Otvos J, Sanchez OA, Mackey RH, Tracy R, Jacobs DR Jr. Comparison of the predictive value of GlycA and other biomarkers of inflammation for total death, incident cardiovascular events, noncardiovascular and noncancer inflammatory-related events, and total cancer events. Clin Chem. 2016;62:1020-31.

18. Akinkuolie AO, Glynn RJ, Padmanabhan L, Ridker PM, Mora S. Circulating $\mathrm{N}$-linked glycoprotein side-chain biomarker, rosuvastatin therapy, and 
incident cardiovascular disease: an analysis from the JUPITER trial. J Am Heart Assoc. 2016;5(7):e003822.

19. Akinkuolie AO, Pradhan AD, Buring JE, Ridker PM, Mora S. Novel protein glycan side-chain biomarker and risk of incident type 2 diabetes mellitus. Arterioscler Thromb Vasc Biol. 2015;35:1544-50.

20. Connelly MA, Gruppen EG, Wolak-Dinsmore J, Matyus SP, Riphagen IJ, Shalaurova I, Bakker SJ, Otvos JD, Dullaart RP. GlycA, a marker of acute phase glycoproteins, and the risk of incident type 2 diabetes mellitus: PREVEND study. Clin Chim Acta. 2016;452:10-7.

21. Bartlett DB, Connelly MA, AbouAssi H, Bateman LA, Tune KN, Huebner JL, Kraus VB, Winegar DA, Otvos JD, Kraus WE, Huffman KM. A novel inflammatory biomarker, GlycA, associates with disease activity in rheumatoid arthritis and cardio-metabolic risk in BMI-matched controls. Arthritis Res Ther. 2016;18:86.

22. Chung CP, Ormseth MJ, Connelly MA, Oeser A, Solus JF, Otvos JD, Raggi $P$, Stein CM. GlycA, a novel marker of inflammation, is elevated in systemic lupus erythematosus. Lupus. 2016;25:296-300,

23. Durcan L, Winegar DA, Connelly MA, Otvos JD, Magder LS, Petri M. Longitudinal evaluation of lipoprotein variables in systemic lupus erythematosus reveals adverse changes with disease activity and prednisone and more favorable profiles with hydroxychloroquine therapy. J Rheumatol. 2016:43:745-50

24. Joshi AA, Lerman JB, Aberra TM, Afshar M, Teague HL, Rodante JA, Krishnamoorthy P, Ng Q, Aridi TZ, Salahuddin T, et al. GlycA is a nove biomarker of inflammation and subclinical cardiovascular disease in psoriasis. Circ Res. 2016:119:1242-53.

25. Solomon DH, Karlson EW, Rimm EB, Cannuscio CC, Mandl LA, Manson JE, Stampfer MJ, Curhan GC. Cardiovascular morbidity and mortality in women diagnosed with rheumatoid arthritis. Circulation. 2003;107:1303-7.

26. Turesson C, Jarenros A, Jacobsson L. Increased incidence of cardiovascular disease in patients with rheumatoid arthritis: results from a community based study. Ann Rheum Dis. 2004;63:952-5.

27. Avina-Zubieta JA, Choi HK, Sadatsafavi M, Etminan M, Esdaile JM, Lacaille D. Risk of cardiovascular mortality in patients with rheumatoid arthritis: a meta-analysis of observational studies. Arthritis Rheum. 2008;59:1690-7.

28. Meune C, Touze E, Trinquart L, Allanore Y. Trends in cardiovascular mortality in patients with rheumatoid arthritis over 50 years: a systematic review and meta-analysis of cohort studies. Rheumatol. 2009;48:1309-13.

29. Meune C, Touze E, Trinquart L, Allanore Y. High risk of clinical cardiovascular events in rheumatoid arthritis: levels of associations of myocardial infarction and stroke through a systematic review and meta-analysis. Arch Cardiovasc Dis. 2010;103:253-61.

30. Armstrong AW, Harskamp CT, Armstrong EJ. The association between psoriasis and hypertension: a systematic review and meta-analysis of observational studies. J Hypertens. 2013;31:433-42.

31. Raaby L, Ahlehoff O, de Thurah A. Psoriasis and cardiovascular events: updating the evidence. Arch Dermatol Res. 2017;309:225-8.

32. Myasoedova E, Crowson CS, Kremers HM, Roger VL, Fitz-Gibbon PD, Therneau TM, Gabriel SE. Lipid paradox in rheumatoid arthritis: the impact of serum lipid measures and systemic inflammation on the risk of cardiovascular disease. Ann Rheum Dis. 2011;70:482-7.

33. Choy E, Ganeshalingam K, Semb AG, Szekanecz Z, Nurmohamed M Cardiovascular risk in rheumatoid arthritis: recent advances in the understanding of the pivotal role of inflammation, risk predictors and the impact of treatment. Rheumatology (Oxford). 2014;53:2143-54.

34. Bag-Ozbek A, Giles JT. Inflammation, adiposity, and atherogenic dyslipidemia in rheumatoid arthritis: is there a paradoxical relationship? Curr Allergy Asthma Rep. 2015;15:497.

35. Alemao E, Cawston H, Bourhis F, Al M, Rutten-van Molken M, Liao KP, Solomon DH. Comparison of cardiovascular risk algorithms in patients with vs without rheumatoid arthritis and the role of C-reactive protein in predicting cardiovascular outcomes in rheumatoid arthritis. Rheumatol. 2017;56(5):777-86.

36. Chandler PD, Akinkuolie AO, Tobias DK, Lawler PR, Li C, Moorthy MV, Wang L, Duprez DA, Jacobs DR, Glynn RJ, et al. Association of N-linked glycoprotein acetyls and colorectal cancer incidence and mortality. PLoS ONE. 2016;11:e0165615.

37. Clark GH, Fraser CG. Biological variation of acute phase proteins. Ann Clin Biochem. 1993;30(Pt 4):373-6.

38. Koenig W, Sund M, Frohlich M, Lowel H, Hutchinson WL, Pepys MB. Refinement of the association of serum C-reactive protein concentration and coronary heart disease risk by correction for within-subject variation over time: the Monica Augsburg studies, 1984 and 1987. Am J Epidemiol. 2003;158:357-64.

39. DeGoma EM, French B, Dunbar RL, Allison MA, Mohler ER 3rd, Budoff MJ. Intraindividual variability of C-reactive protein: the Multi-Ethnic Study of Atherosclerosis. Atherosclerosis. 2012;224:274-9.

40. Bogaty P, Dagenais GR, Joseph L, Boyer L, Leblanc A, Belisle P, Brophy JM. Time variability of $C$-reactive protein: implications for clinical risk stratification. PLoS ONE. 2013;8:e60759.

41. Muhlestein JB, Mays H, Galenko O, Knowlton K, Otvos JD, Connelly MA, Lappe $D$, Anderson J. Both the novel NMR biomarker of inflammation, GlycA, and hs-CRP are independent and additive predictors of future cardiovascular events among patients undergoing coronary angiography: results from the Intermountain Heart Collaborative Study. J Amer Coll Cardiol. 2017;69(11):1069.

42. Gabay C, Kushner I. Acute-phase proteins and other systemic responses to inflammation. N Engl J Med. 1999;340:448-54.

43. Connelly MA, Winegar DW, Shalaurova I, Otvos JD. Nuclear magnetic resonance measured serum biomarkers and type 2 diabetes risk stratification. J Diab Metab Disord Contr. 2015;2(4):000050.

44. Soininen P, Kangas AJ, Wurtz P, Suna T, Ala-Korpela M. Quantitative serum nuclear magnetic resonance metabolomics in cardiovascular epidemiology and genetics. Circ Cardiovasc Genet. 2015;8:192-206.

45. Mokkala K, Pellonpera O, Roytio H, Pussinen P, Ronnemaa T, Laitinen K. Increased intestinal permeability, measured by serum zonulin, is associated with metabolic risk markers in overweight pregnant women. Metabolism. 2017;69:43-50.

46. Fischer K, Kettunen J, Wurtz P, Haller T, Havulinna AS, Kangas AJ, Soininen P, Esko T, Tammesoo ML, Magi R, et al. Biomarker profiling by nuclear magnetic resonance spectroscopy for the prediction of allcause mortality: an observational study of 17,345 persons. PLoS Med. 2014;11:e1001606.

47. Ridker PM, Everett BM, Thuren T, MacFadyen JG, Chang WH, Ballantyne C, Fonseca F, Nicolau J, Koenig W, Anker SD, et al. Antiinflammatory therapy with canakinumab for atherosclerotic disease. N Engl J Med. 2017;377:1119-31.

\section{Submit your next manuscript to BioMed Central and we will help you at every step:}

- We accept pre-submission inquiries

- Our selector tool helps you to find the most relevant journal

- We provide round the clock customer support

- Convenient online submission

- Thorough peer review

- Inclusion in PubMed and all major indexing services

- Maximum visibility for your research

Submit your manuscript at www.biomedcentral.com/submit
(OioMed Central 